\title{
Design of All-Accelerometer Inertial Measurement Unit for Tremor Sensing in Hand-held Microsurgical Instrument
}

\author{
Wei Tech Ang, Pradeep K. Khosla, and Cameron N. Riviere \\ The Robotics Institute \\ Carnegie Mellon University \\ Pittsburgh, PA 15213 \\ USA
}

\begin{abstract}
We present the design of an all-accelerometer inertial measurement unit (IMU). The IMU forms part of an intelligent hand-held microsurgical instrument that senses its own motion, distinguishes between hand tremor and intended motion, and compensates in real-time the erroneous motion. The new IMU design consists of three miniature dual-axis accelerometers, two of which are housed in a sensor suite at the distal end of the instrument handle, and one located at the proximal end close to the instrument tip. By taking the difference between the accelerometer readings, we decouple the inertial and gravitational accelerations from the rotation-induced (centripetal and tangential) accelerations, hence simplifies the kinematic computation of angular motions. We have shown that the error variance of the Euler orientation parameters $\theta_{x}, \theta_{y}$ and $\theta_{z}$ is inversely proportional to the square of the distance between the three sensor locations. Comparing with a conventional three gyros and three accelerometers IMU, the proposed design reduces the standard deviation of the estimates of translational displacements by $29.3 \%$ in each principal axis and those of the Euler orientation parameters $\theta_{x}, \theta_{y}$ and $\theta_{z}$ by $99.1 \%$, $\mathbf{9 9 . 1 \%}$ and $\mathbf{9 2 . 8 \%}$ respectively.
\end{abstract}

\section{INTRODUCTION}

In microsurgery, surgeons must perform manipulation tasks that approach the limits of human positioning accuracy. The envelope of human manipulation precision is bounded by involuntary hand movements. The best-known erroneous hand motion is physiological tremor. Tremor is defined as any involuntary, approximately rhythmic, and roughly sinusoidal movement [1]. In ophthalmological microsurgery, its significant component is found to be an oscillation at 8-12 $\mathrm{Hz}$ whose frequency is independent of the mechanical properties of the hand and arm [1], and with amplitude of about $50 \mu \mathrm{m}$ peak-to-peak or less $[2,3]$. These inherent limitations complicate many delicate surgical procedures, and make some types of intervention impossible. Examples of delicate procedures include removal of membranes as thin as $20 \mu \mathrm{m}$ from the retina, and operating on tumors in close proximity to crucial brain tissues.

In previous work we have developed an intelligent hand-held microsurgical instrument that senses its own motion, distinguishes tremor from the intended motion of the surgeon, and deflects the instrument tip in realtime with an equal but opposite motion in order to compensate the erroneous movement [4]. The first prototype is equipped with a standard IMU, containing three miniature rate gyros (Tokin CG-16D) and three accelerometers (Crossbow CXL02LF3 tri-axial), at the distal end of the instrument handle from the instrument tip.

The key problem of this conventional IMU design is that the orientation estimation depends totally on the noisy low-cost gyros. The noisy gyros harm the instrument tip displacement estimation in two ways: (i) Any angle estimation errors at the distal end are translated into a magnified displacement error at the instrument tip; (ii) Errors in orientation estimation cause errors in gravity compensation in the accelerometer readings and in turn corrupt the tip displacement estimation.

High quality gyros with the precision needed for this application are too bulky to be fitted into a hand-held device [5, 6], and they are almost always too costly [7]. On the other hand, inexpensive, batch-processed gyros, although having suitable size and weight, currently lack the level of required precision. Technology breakthrough in the gyro manufacturing process is not likely in the near future due to challenges associated with microminiaturization of gyros [8]. Exploiting the more affordable and mature micro-machined accelerometer technology, researchers have proposed gyro-free or allaccelerometer sensor designs. Chen et al. [9] proposed an original cube configuration design with six accelerometers, the minimum number of sensors to recover all the six kinematic parameters. However, no comparison with the conventional three gyros three accelerometers design has been reported.

We propose in this paper a novel IMU design using three dual-axis accelerometers and no gyros. Section II gives a brief overview of the intelligent microsurgical instrument to provide more insight to the background of the problem. It also describes the design of the proposed new inertial measurement unit and discusses the motivations for choosing this configuration. Section III derives the motion equations of the instrument from a differential kinematics perspective that decouples rotation-induced accelerations from inertial accelerations and gravity induced accelerations. In Section IV, error covariance analysis is performed on the proposed design, and compared to the standard three-gyro, threeaccelerometer configuration. 


\section{DESIGN OF INERTIAL MEASUREMENT UNIT}

The intelligent microsurgical instrument is made up of three sub-systems: the sensing system, the filtering algorithms, and the tip manipulator system. An overview of the system is shown in Fig. 1.

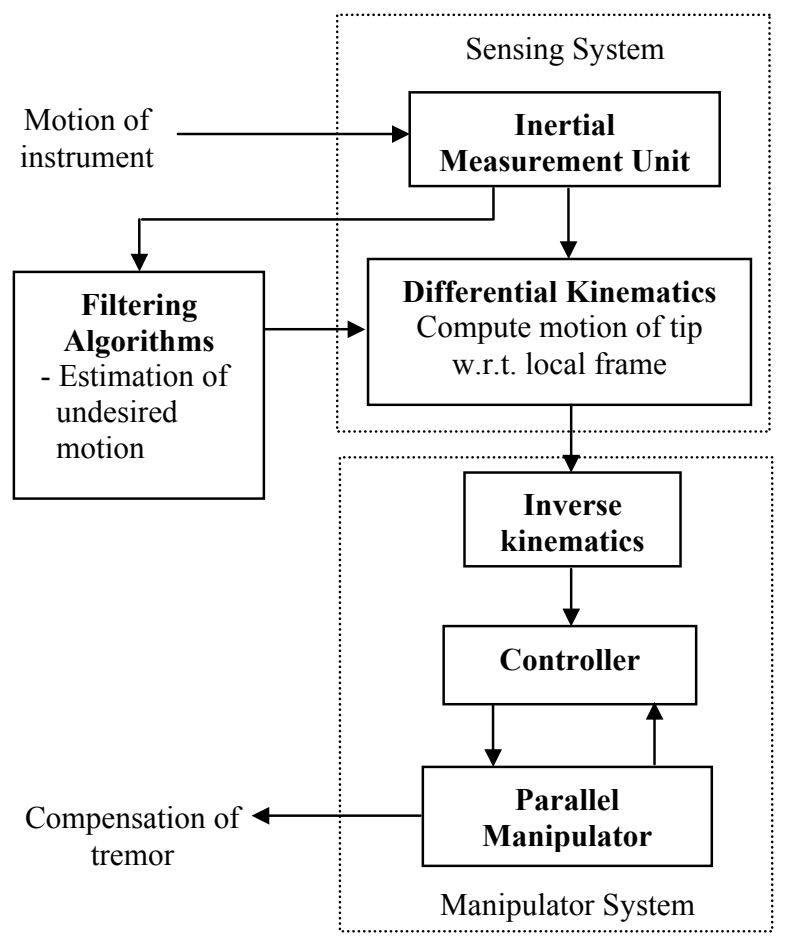

Fig.1 System overview of the intelligent microsurgical instrument for tremor compensation

The inertial measurement unit senses the motion of the instrument and feeds the raw sensory information to the filtering algorithms. The filtering algorithms remove the intended motion, compensate for the phase shift due to analog and digital filters, and model and estimate the tremor based on a dynamic sinusoidal model [10]. The motion of the instrument tip is then computed by forward kinematics after removal of the gravity. Next, the manipulator system deflects the tip in real-time in an opposite direction but equal magnitude to the motion due to hand tremor. Only the design of the inertial measurement unit will be discussed in this paper.

The inertial measurement unit for our hand-held microsurgical instrument consists of a total of three miniature Analog Devices ADXL-202E dual-axis accelerometers (dimensions: $5 \mathrm{~mm} \times 5 \mathrm{~mm} \times 2 \mathrm{~mm}$, weight: $<1 \mathrm{~g}$ ). These sensors are housed in two locations in the instrument handle, as shown in Fig. 2. The sensor suite at the back end of the instrument houses two dualaxis accelerometers in orthogonal orientations (two $X$-, one $Y$-, and one $Z$-sensing directions). One dual-axis accelerometer is located at the front end close to the intraocular shaft, measuring motion in $X$ and $Y$ directions.

There are a few reasons for adopting this design for the sensing system. Firstly, in our application of sensing and compensating erroneous hand movement during microsurgery, the internally referenced inertial sensing technology emerges to be the most suited. From a technical standpoint, it meets all mandatory technical specifications (resolution and accuracy: $<1 \mu \mathrm{m}$, system bandwidth: $>15 \mathrm{~Hz}$ ). More importantly, from an end-user perspective, all externally referenced sensors (E.g. Optotrak and Polaris, Northern Digital Inc.; Fastrak, Polhemus, Ultratrak, Polhemus Inc.; Constellation, Intersense Inc.), require a line of sight or other similar provision and may be a hindrance to the way the surgery is performed, given the tight workspace the surgeon has to work with. Moreover, in our attempt to sense and compensate hand tremor, the signal of interest becomes the higher frequency hand tremor, while the intended motion becomes the "noise," as it were. When the filtering algorithm separates the slower varying voluntary motion from the tremor, the low-frequency sensor drifts are removed at the same time. As a result, precise tracking of the absolute pose of the instrument is not of importance, as long as we can remove gravity from the kinematic model to recover the tremor motion.

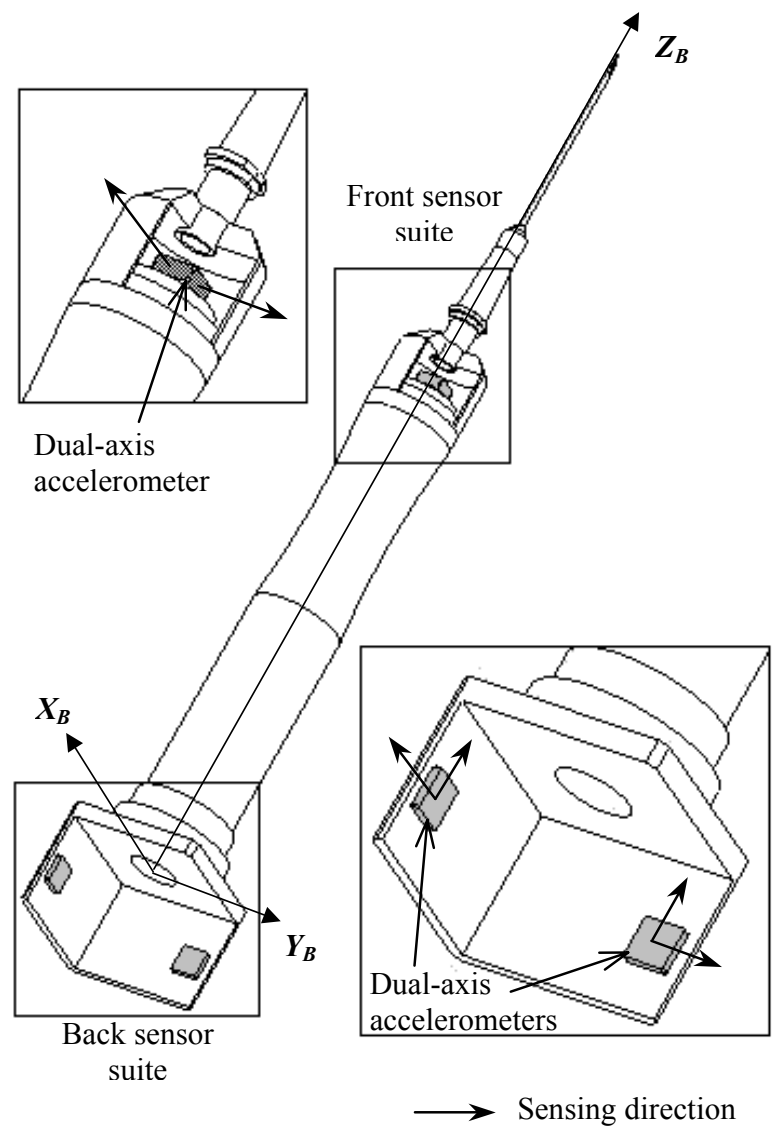

Fig. 2. Design of the inertial measurement unit. 
Secondly, miniature accelerometers are more superior to miniature low-cost gyros, in terms of noise, linearity and accuracy. Thirdly, compared to other existing allaccelerometer IMU in a cube configuration, the proposed design can use off-the-shelf dual-axis accelerometers, since the sensing directions can be orthogonal. This results in a more compact, simpler, and cheaper design. Fourthly, having six accelerometers provides one degree of sensing redundancy in each of the three translational DOF and enables the error covariances to be reduced by techniques such as Kalman filtering. Last but not least, the most compelling reason to have an IMU design with accelerometers placed apart from each other is that this configuration enables high quality sensing, to be shown in the next sections.

\section{DIFFERENTIAL SENSING KINEMATICS}

We define a body frame $\{B\}$ at the base of back sensor suite, with its $Z_{B}$-axis collinear with the instrument tip as shown in Fig 3.

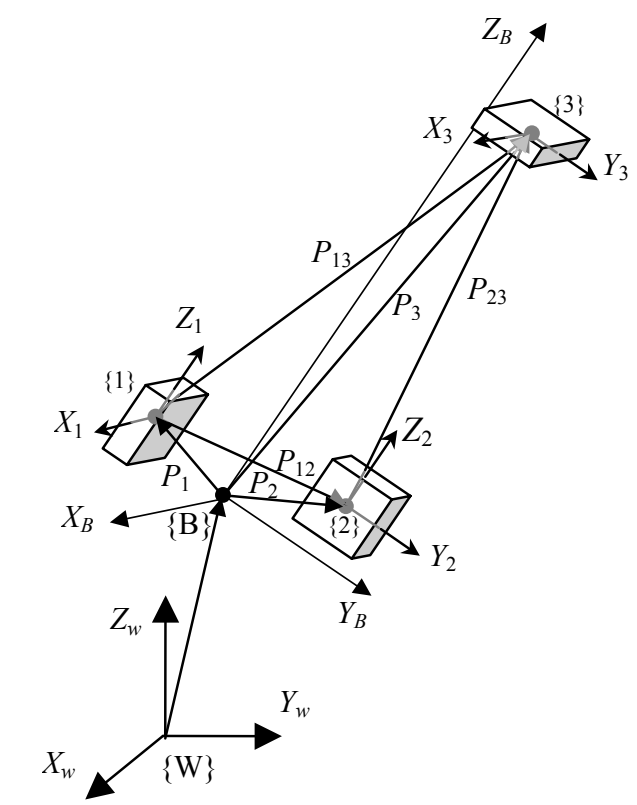

Fig. 3 Kinematic representation of microsurgical instrument

In the back sensor suite, there are two dual axis accelerometers, ${ }^{B} A_{1}=\left[{ }^{B} a_{1 x} \bullet{ }^{B} a_{1 z}\right]^{\mathrm{T}}$ and ${ }^{B} A_{2}=\left[\bullet^{B} a_{2 y}\right.$ $\left.{ }^{B} a_{1 z}\right]^{\mathrm{T}}$. The third dual axis accelerometers, ${ }^{B} A_{3}=\left[{ }^{B} a_{3 x}{ }^{B} a_{3 y}\right.$ - $]^{\mathrm{T}}$ is located at the front sensor suite close to the instrument tip. The symbol $\bullet$ means undefined. The locations of the accelerometers with respect to the body frame $\{B\}$ are known from the mechanical design and through sensor calibrations. Without loss of generality, we assume that the proof masses of each of the dual axis accelerometer are coincident at $\{1\},\{2\}$ and $\{3\}$ respectively, and all the sensing axes are aligned with the body principle axes: $X_{B}, Y_{B}$, and $Z_{B}$. Fig. $3.1 \mathrm{~b}$ shows the accelerometer locations, the sensing axes and kinematic frames of the instrument.

The $X_{B}, Y_{B}$ and $Z_{B}$ axes of frame $\{\mathrm{B}\}$ are initialized to coincide with $X_{\mathrm{W}}, Y_{\mathrm{W}}$ and $Z_{\mathrm{W}}$ axes of the world coordinate system $\{\mathrm{W}\}$ at time $t=0$. To derive the angular velocity vector, ${ }^{B} \Omega=\left[{ }^{B} \omega_{x}{ }^{B} \omega_{y}{ }^{B} \omega_{z}\right]^{\mathrm{T}}$ from the accelerometers, we start from the motion equation of a rigid body in $\mathbf{E}^{3}$ space.

The total accelerations, ${ }^{B} A_{i}$, each accelerometer at $\{i\}$ senses include the inertial acceleration of the body, ${ }^{B} A_{C G}$, the gravity, ${ }^{B} g$, and the rotation-induced accelerations: the centripetal acceleration, ${ }^{B} A_{i / C}$, and the tangential acceleration, ${ }^{B} A_{i / T}$,

$$
\begin{aligned}
& { }^{B} A_{i}={ }^{B} A_{C G}+{ }^{B} g+{ }^{B} A_{i / C}+{ }^{B} A_{i / T}, i=1,2,3 ; \\
& { }^{B} A_{i}={ }^{B} A_{C G}+{ }^{B} g+\underbrace{B}_{\text {rotation-induced }} \Omega \times{ }^{B} \Omega \times R+{ }^{B} \alpha \times R
\end{aligned}
$$

where the $R$ is the vector from the unknown instantaneous center of rotation to the point of sensing.

Taking the difference between the accelerations readings at $\{1\},\{2\}$ and $\{3\}$, the non-rotation induced acceleration components ${ }^{B} A_{C G}$ and ${ }^{B} g$ are eliminated, since the linear inertial acceleration of the body and the gravity should be identical at different locations,

$$
\begin{aligned}
{ }^{B} A_{13} & ={ }^{B} A_{3}-{ }^{B} A_{1}={ }^{B} \Omega \times{ }^{B} \Omega \times{ }^{B} P_{13}+{ }^{B} \alpha \times{ }^{B} P_{13} \\
& =\left[{ }^{B} a_{13 x} \bullet \bullet\right]^{\mathrm{T}} \\
{ }^{B} A_{23} & ={ }^{B} A_{3}-{ }^{B} A_{2}={ }^{B} \Omega \times{ }^{B} \Omega \times{ }^{B} P_{23}+{ }^{B} \alpha \times{ }^{B} P_{23} \\
& =\left[\bullet{ }^{B} a_{23 y} \bullet\right]^{\mathrm{T}} \\
{ }^{B} A_{12} & ={ }^{B} A_{2}-{ }^{B} A_{1}={ }^{B} \Omega \times{ }^{B} \Omega \times{ }^{B} P_{12}+{ }^{B} \alpha \times{ }^{B} P_{12} \\
& =\left[\bullet \bullet{ }^{B} a_{12 z}\right]^{\mathrm{T}}
\end{aligned}
$$

where the symbol $\bullet$ denoted undefined quantity and ${ }^{B} P_{i j}=$ $\left[{ }^{B} p_{i j x}{ }^{B} p_{i j y}{ }^{B} p_{i j z}\right]^{\mathrm{T}}, i, j=1,2,3$, is position vector from $\{i\}$ to $\{j\}$ with respect to $\{\mathrm{B}\}$, which are known values from the hardware design and calibrations. The differential velocity at the location $\{i\}$ at time $t$ is given by integrating (2),

$$
{ }^{B} U_{i j}(t)=\int{ }^{B} A_{i j}(t) d t+{ }^{B} U_{i j}(t-T), i, j=1,2,3 ;
$$

where $T$ is the sampling period.

Taking only the defined dimensions in (2a-c) and solving the simultaneous equations yield the angular velocity vector,

$$
{ }^{B} \Omega_{A}(t)=P_{D}{ }^{-1} U_{D}=\left[{ }^{B} \omega_{A x}{ }^{B} \omega_{A y}{ }^{B} \omega_{A z}\right]^{\mathrm{T}},
$$

where

$$
P_{D}=\left[\begin{array}{ccc}
0 & { }^{B} p_{13 z} & -{ }^{B} p_{13 y} \\
-{ }^{B} p_{23 z} & 0 & { }^{B} p_{23 x} \\
{ }^{B} p_{12 y} & -{ }^{B} p_{13 x} & 0
\end{array}\right] ; U_{D}=\left[\begin{array}{c}
{ }^{B} u_{13 x} \\
{ }^{B} u_{23 y} \\
{ }^{B} u_{12 z}
\end{array}\right]
$$

The angular displacement vector is then given by

$$
{ }^{B} \Theta(t)=\int{ }^{B} \Omega(t) d t+{ }^{B} \Theta(t-T)
$$


The directional cosine matrix that relates the body frame $\{B\}$ to the world coordinate system $\{W\}$ at each sampling interval is updated by first updating the quaternions,

$$
\begin{aligned}
\dot{q}(t) & =\widetilde{\Omega}(t) q(t) \\
q(t) & =\int \dot{q}(t) d t+q(t-T) \\
\widetilde{\Omega} & =\frac{1}{2}\left[\begin{array}{ccc:c}
0 & \omega_{z} & -\omega_{y} & \omega_{x} \\
-\omega_{z} & 0 & \omega_{x} & \omega_{y} \\
\omega_{y} & -\omega_{x} & 0 & \omega_{z} \\
\hdashline-\omega_{x} & -\omega_{y} & -\omega_{z} & 0
\end{array}\right]
\end{aligned}
$$

where $\widetilde{\Omega}$ is the augmented cross product matrix. In terms of quaternions, the directional cosine matrix is

$$
\begin{aligned}
& { }^{W} C_{B}= \\
& {\left[\begin{array}{ccc}
q_{0}^{2}+q_{1}^{2}-q_{2}^{2}-q_{3}^{2} & 2\left(q_{1} q_{2}-q_{0} q_{3}\right) & 2\left(q_{1} q_{3}+q_{0} q_{2}\right) \\
2\left(q_{1} q_{2}+q_{0} q_{3}\right) & q_{0}^{2}-q_{1}^{2}+q_{2}^{2}-q_{3}^{2} & 2\left(q_{2} q_{3}-q_{0} q_{1}\right) \\
2\left(q_{1} q_{3}-q_{0} q_{2}\right) & 2\left(q_{2} q_{3}+q_{0} q_{1}\right) & q_{0}^{2}-q_{1}^{2}-q_{2}^{2}+q_{3}^{2}
\end{array}\right]}
\end{aligned}
$$

The effective translational body motion with respect to $\{\mathrm{W}\}$ is obtained from removing the gravity component from the accelerometer readings,

$$
{ }^{W} A_{i E}={ }^{W} C_{B}{ }^{B} A_{i}-{ }^{W} g
$$

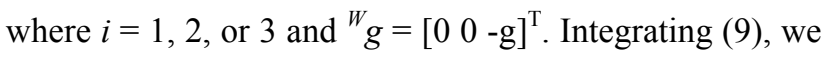
get the effective velocities at $\{i\}$,

$$
{ }^{W} U_{i E}(t)=\int{ }^{W} A_{i E}(t) d t+{ }^{W} U_{i E}(t-T)
$$

The effective velocity at the origin of $\{B\}$ with respect to $\{\mathrm{W}\},{ }^{W} U_{E}=\left[{ }^{W} u_{E x}{ }^{W} u_{E y}{ }^{W} u_{E z}\right]^{\mathrm{T}}$ is found by transforming the position and angular velocity vectors into $\{\mathrm{W}\}$,

$$
\begin{aligned}
& { }^{W} P_{i B}(t)={ }^{W} C_{B}(t)\left(-{ }^{B} P_{i}\right), \\
& { }^{W} \Omega(t)={ }^{W} C_{B}(t)^{B} \Omega(t)
\end{aligned}
$$

and then compound the results derived from different accelerometers by their respective error variances,

$$
\begin{aligned}
{ }^{W} u_{E x}= & \Sigma_{A 13 x}\left({ }^{W} u_{1 x}+{ }^{W} p_{1 B z}{ }^{W} \omega_{A y}-{ }^{W} p_{1 B y}{ }^{W} \omega_{A z}\right)+ \\
& \Sigma_{A 31 x}\left({ }^{W} u_{3 x}+{ }^{W} p_{3 B z}{ }^{W} \omega_{A y}-{ }^{W} p_{3 B y}{ }^{W} \omega_{A z}\right) \\
{ }^{W} u_{E y}= & \Sigma_{A 23 y}\left({ }^{W} u_{2 y}+{ }^{W} p_{2 B x}{ }^{W} \omega_{A z}-{ }^{W} p_{2 B z}{ }^{W} \omega_{A x}\right)+ \\
& \Sigma_{A 32 y}\left({ }^{W} u_{3 y}+{ }^{W} p_{3 B x}{ }^{W} \omega_{A z}-{ }^{W} p_{3 B z}{ }^{W} \omega_{A x}\right) \\
{ }^{W} u_{E z}= & \Sigma_{A 12 z}\left({ }^{W} u_{1 z}+{ }^{W} p_{1 B y}{ }^{W} \omega_{A x}-{ }^{W} p_{1 B x}{ }^{W} \omega_{A y}\right)+ \\
& \Sigma_{A 21 z}\left({ }^{W} u_{2 z}+{ }^{W} p_{2 B y}{ }^{W} \omega_{A x}-{ }^{W} p_{2 B x}{ }^{W} \omega_{A y}\right)
\end{aligned}
$$

where $\Sigma_{A j i d}=\sigma_{\text {Aid }}^{2} /\left(\sigma_{\text {Aid }}^{2}+\sigma_{\text {Ajd }}^{2}\right), d=x, y$, or $z$.

To obtain the instantaneous velocity the instrument tip with respect $\{\mathrm{W}\}$,

$$
{ }^{W} U_{\text {tip }}(t)={ }^{W} U_{E}(t)+{ }^{W} \Omega(t) \times{ }^{W} C_{B}(t)^{B} P_{t i p}
$$

where the tip position with respect to the body frame $\{\mathrm{B}\}$ is ${ }^{B} P_{\text {tip }}=\left[\begin{array}{lll}0 & 0 & l_{1}\end{array}\right]^{\mathrm{T}}$. Integrating (14) we get the instantaneous tip displacement

$$
{ }^{W} P_{\text {tip }}(t)=\int{ }^{W} U_{t i p}(t) d t+{ }^{W} P_{t i p}(t-T)
$$

\section{ERROR COVARIANCE COMPARISON OF IMU DESIGNS}

In this section, we show the superiority of the proposed IMU design over the conventional three gyros three accelerometers design. We do so by comparing the error covariance in the estimated angular and translational displacement of the instrument.

Since we have two accelerometers in each sensing axis, compounding the redundant sensing information results in an improvement of $29.3 \%$ or a factor of $\sqrt{2}$ in the standard deviations of the translational displacement measurements, assuming all accelerometers have identical noise characteristics.

$$
\sigma_{A}=\sqrt{\frac{\sigma_{A i}^{2}}{\sigma_{A i}^{2}+\sigma_{A j}^{2}}}=\frac{\sigma_{A i}}{\sqrt{2}}, \text { for } \sigma_{A i}^{2}=\sigma_{A j}^{2} .
$$

To analyze the error variance of the angular displacement estimations, we first define the sensor vectors, $G=\left[\begin{array}{lll}\omega_{x} & \omega_{y} & \omega_{z}\end{array}\right]^{\mathrm{T}}$ and $A=\left[\begin{array}{lllll}a_{1 x} & a_{1 z} & a_{2 y} & a_{2 z} & a_{3 x}\end{array}\right.$ $\left.a_{3 y}\right]^{\mathrm{T}}$. Taking into account the stochasticity of the sensors, the sensor vectors become

$$
A=\bar{A}+\delta A ; G=\bar{G}+\delta G
$$

where $\bar{A}$ and $\bar{G}$ are the means, $\delta A$ and $\delta G$ are zero mean random vectors with known covariances,

$$
\begin{aligned}
& \mathrm{C}(A)_{6 \times 6}=\mathrm{E}\left[(A-\bar{A})(A-\bar{A})^{\mathrm{T}}\right], \\
& \mathrm{C}(G)_{3 \times 3}=\mathrm{E}\left[(G-\bar{G})(G-\bar{G})^{\mathrm{T}}\right] .
\end{aligned}
$$

Using the variance of the Analog Devices ADXL-202 accelerometers $\left(\sim 3850 \mathrm{~mm}^{2} / \mathrm{s}^{4}\right)$ obtained from empirical data, and using $T=1 \mathrm{~ms}$, the variance per sampling period of $d_{x}, d_{y}$ and $d_{z}$ are computed and tabulated in Table I.

The Euler angles, $\Theta_{G}=\left[\theta_{G x} \theta_{G y} \theta_{G z}\right]^{\mathrm{T}}$, from the gyros are obtained from direct integration of the sensed angular velocity. For simplicity in comparison, we use a trapezoidal integration rule:

$$
\begin{aligned}
& \Theta_{G}=0.5 \cdot \mathrm{T} \cdot(G[t+1]+G[t]) \\
& \mathrm{C}\left(\Theta_{G}\right)=0.25 \cdot \mathrm{T}^{2} \cdot(\mathrm{C}(G[t+1])+\mathrm{C}(G[t]))
\end{aligned}
$$

Using the variance of the Tokin CG-16D gyros $(\sim 2.0$ $\mathrm{deg}^{2} / \mathrm{s}^{2}$ ) obtained from empirical data, and a sampling time of $\mathrm{T}=1 \mathrm{~ms}$, the individual variance or the diagonal elements of the covariance matrix $\mathrm{C}\left(\Theta_{G}\right)$ are computed and tabulated in Table I.

From Section III, vector $\Theta_{A}$ and the accelerometer vector $A$ are related by vector function $f$, 


$$
\Theta_{\mathrm{A}}=f(A)=f(\bar{A}+\delta A)
$$

Taking the Taylor Series expansion [11],

$$
f(\bar{A}+\delta A)=f(\bar{A})+\nabla f \delta A+1 / 2 \nabla^{2} f \delta A^{2}+\ldots
$$

where $\nabla^{i} f \cdot \delta A^{i}$ is the informal notation for the $i^{\text {th }}$ order term in the multidimensional Taylor Series and $\nabla f$ is the Jacobian of $f$ evaluated at $\bar{A}$.

$$
\nabla f=\frac{\partial f(A)}{\partial A}(\bar{A})=\left[\begin{array}{cccc}
\frac{\partial f_{1}}{\partial a_{1 x}} & \frac{\partial f_{1}}{\partial a_{1 y}} & \cdots & \frac{\partial f_{1}}{\partial a_{3 x}} \\
\frac{\partial f_{2}}{\partial a_{1 x}} & \frac{\partial f_{2}}{\partial a_{1 y}} & \cdots & \frac{\partial f_{2}}{\partial a_{3 x}} \\
\vdots & \vdots & \ddots & \vdots \\
\frac{\partial f_{6}}{\partial a_{1 x}} & \frac{\partial f_{6}}{\partial a_{1 y}} & \cdots & \frac{\partial f_{6}}{\partial a_{3 x}}
\end{array}\right]_{A=\bar{A}}
$$

The covariance of $\Theta_{A}$ up to the first order is then

$$
\mathrm{C}\left(\Theta_{\mathrm{A}}\right) \approx \nabla f C(A)(\nabla f)^{\mathrm{T}}+\ldots
$$

Performing a design optimization, we arrivev at a design configuration of ${ }^{B} P_{12}=\left[\begin{array}{lll}-d_{1} & d_{1} & 0\end{array}\right]^{\mathrm{T}},{ }^{B} P_{23}=\left[\begin{array}{ll}d_{3}-d_{3} \\ d_{2}\end{array}\right.$ $\left.d_{2}\right]^{\mathrm{T}}$, and ${ }^{B} P_{13}=\left[-d_{3} d_{3} d_{2}\right]^{\mathrm{T}}$. Using $T=1 \mathrm{~ms}$, the variance per sampling period of $\theta_{A x}, \theta_{A y}$ and $\theta_{A z}$ are

$$
\begin{gathered}
\sigma_{\theta A x}^{2}=\sigma_{\theta A y}^{2}=2.76 \times 10^{-8}\left(\frac{1}{2 d_{1}^{2}}+\frac{1}{d_{2}^{2}}\right) \mathrm{deg}^{2}, \\
\sigma_{\theta A z}^{2}=2.76 \times 10^{-8}\left(\frac{1}{d_{3}^{2}}+\frac{1}{2}\left(\frac{d_{2}}{d_{1} d_{3}}\right)^{2}\right) \mathrm{deg}^{2} ;
\end{gathered}
$$

Considering the physical constraints of a handheld device, we design the sensor positions to be $d_{1}=20 \mathrm{~mm}$, $d_{2}=100 \mathrm{~mm}$, and $d_{3}=12 \mathrm{~mm}$. With this design, the results of (26a-b) are tabulated in Table I.

TABLE I

Comparison of error variance and standard deviations of translational and angular measurements by a conventional three gyros three accelerometers (3G-3A) design versus the proposed all-accelerometers (6A) design.

\begin{tabular}{|l|l|c|c|c|}
\hline \multicolumn{2}{|c|}{ Measurement } & $\theta_{x}, \theta_{y}$ & $\theta_{z}$ & $d_{x}, d_{y}, d_{z}$ \\
\hline $\begin{array}{l}\text { Error } \\
\text { Var., } \\
\sigma^{2}\end{array}$ & $3 \mathrm{G}-3 \mathrm{~A}$ & $\begin{array}{c}1 \times 10^{-6} \\
\mathrm{deg}^{2}\end{array}$ & $\begin{array}{c}1 \times 10^{-6} \\
\mathrm{deg}^{2}\end{array}$ & $\begin{array}{c}1.54 \times 10^{-8} \\
\mathrm{~mm}^{2}\end{array}$ \\
\cline { 2 - 5 } & $6 \mathrm{~A}$ & $\begin{array}{c}7.44 \times 10^{-11} \\
\mathrm{deg}^{2}\end{array}$ & $\begin{array}{c}5.17 \times 10^{-9} \\
\mathrm{deg}^{2}\end{array}$ & $\begin{array}{c}7.7 \times 10^{-9} \\
\mathrm{~mm}^{2}\end{array}$ \\
\hline $\begin{array}{l}\text { Error } \\
\text { Std. } \\
\begin{array}{l}\text { Dev., } \\
\sigma\end{array}\end{array}$ & 3G-3A & $\begin{array}{c}1 \times 10^{-3} \\
\mathrm{deg}\end{array}$ & $\begin{array}{c}1 \times 10^{-3} \\
\mathrm{deg}\end{array}$ & $\begin{array}{c}1.24 \times 10^{-4} \\
\mathrm{~mm}\end{array}$ \\
\cline { 2 - 5 } & $6 \mathrm{~A}$ & $\begin{array}{c}8.63 \times 10^{-6} \\
\mathrm{deg}\end{array}$ & $\begin{array}{c}7.19 \times 10^{-5} \\
\mathrm{deg}\end{array}$ & $\begin{array}{c}8.77 \times 10^{-5} \\
\mathrm{~mm}\end{array}$ \\
\cline { 2 - 5 } & $\begin{array}{l}\text { \% } \\
\text { Reduction }\end{array}$ & $99.1 \%$ & $92.8 \%$ & $29.3 \%$ \\
\hline
\end{tabular}

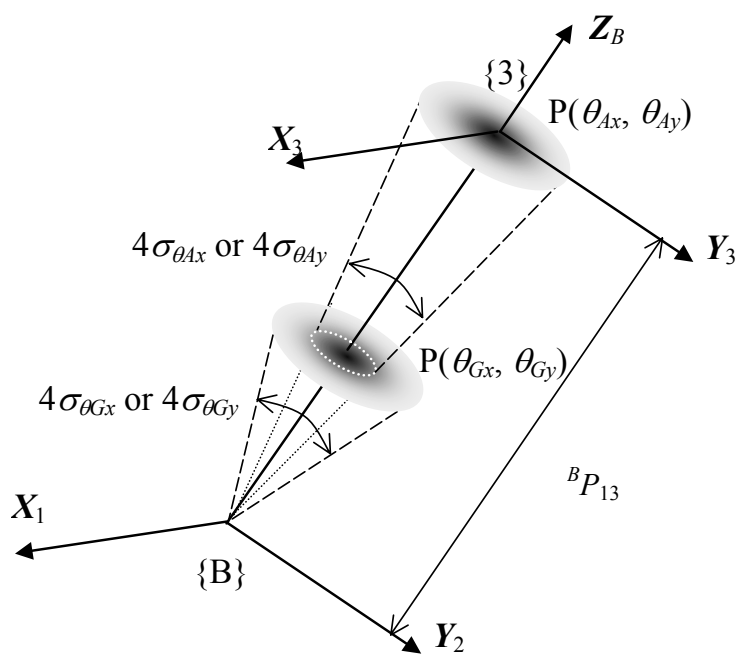

Fig. 4 Comparison of the joint distributions of $\theta_{x}$ and $\theta_{y}$ estimates between an all-gyro $\left(\mathrm{P}\left(\theta_{G x}, \theta_{G y}\right)\right)$ and an allaccelerometer $\left(\mathrm{P}\left(\theta_{A x}, \theta_{A y}\right)\right)$ design. Higher density regions at the centers represent higher probability.

The standard deviation of the $\theta_{A x}, \theta_{A y}$, and $\theta_{A z}$ are reduced by $99.1 \%, 99.1 \%$ and $92.8 \%$ over those of $\theta_{G x}$, $\theta_{G y}$, and $\theta_{G y}$ respectively. The intuition of this result is depicted in Fig. 4, taking $\theta_{G x}$ and $\theta_{G y}$ as examples. The discs labeled $\mathrm{P}\left(\theta_{G x}, \theta_{G y}\right)$ and $\mathrm{P}\left(\theta_{A x}, \theta_{A y}\right)$ are the joint distributions of the $\Theta_{G x y}=\left[\begin{array}{ll}\theta_{G x} & \theta_{G y}\end{array}\right]^{\mathrm{T}}$ and $\Theta_{A x y}=\left[\begin{array}{ll}\theta_{A x} & \theta_{A y}\end{array}\right]^{\mathrm{T}}$ estimates from gyros and accelerometers respectively. Higher density regions at the centers represent higher probability. With the same uncertainty distribution at $\{3\}$, the larger $\left|{ }^{B} P_{13}\right|\left(=\left|{ }^{B} P_{23}\right|\right)$ gets, the smaller the angle sustaining the cone will become. This angle may be viewed as being 4 times the standard deviation $(99.9 \%$ confidence level) of $\theta_{A x}$ or $\theta_{A y}$, since $\sigma_{\theta A x}$ and $\sigma_{\theta A y}$ are identical. The noise in the $\theta_{A z}$ measurement is the worst because it would be impractical to increase the length $\left|{ }^{B} P_{12}\right|$ due to the physical constraints in the handheld instrument.

On top of the $29.3 \%$ direct improvement in the translation sensing, the improvement in the orientation estimation will result in a much complete removal of the gravity, which in turn has a great impact on the quality of the translation sensing since gravity is typically two orders of magnitude larger than hand accelerations.

\section{CONCLUSION}

We have presented the design of an all-accelerometer inertial measurement unit for tremor sensing in a handheld microsurgical instrument. The inertial measuring unit consists of three miniature dual-axis accelerometers, housed in two locations. At the back sensor suite, we have 
two dual-axis accelerometers, with sensing axes: one along ${ }^{B} X$-, one along ${ }^{B} Y$-, and two along ${ }^{B} Z$ axis (the long axis of the instrument). One dual-axis accelerometer is located at the front end close to the instrument tip, measuring motion in ${ }^{B} X$ and ${ }^{B} Y$ directions. With differential sensing, we are able to decouple the rotationinduced motion from the translational motion and the gravity. Comparing with a conventional three gyros and three accelerometers IMU, the proposed design reduces the standard deviation of the estimates of translational displacements by $29.3 \%$ in each principal axis and those of the Euler orientation parameters $\theta_{x}, \theta_{y}$ and $\theta_{z}$ by $99.1 \%$, $99.1 \%$ and $92.8 \%$ respectively.

\section{REFERENCES}

[1] R. J. Elble and W. C. Koller, Tremor. Baltimore: Johns Hopkins, 1990.

[2] C. N. Riviere and P. S. Jensen, "A study of instrument motion in retinal microsurgery," Proc. 21st Annual Intl. Conf. IEEE Eng. Med. Biol. Soc., 2000.

[3] I. W. Hunter, T. D. Doukoglou, S. R. Lafontaine, P. G. Charette, L. A. Jones, M. A. Sagar, G. D. Mallinson, and P. J. Hunter, "A teleoperated microsurgical robot and associated virtual environment for eye surgery," Presence, 2:265-280, 1993.

[4] W.T. Ang, C.N. Riviere, and P.K. Khosla, "Design and Implementation of Active Error Canceling in Hand-held Microsurgical Instrument," Proc. Intl. Conf. Intell. Robot. Syst., Hawaii, 2001, pp.11061111.

[5] N. Barbour and G. Schmidt, "Inertial Sensor Technology Trends," IEEE Sensors Journal, vol.1. No. 4, pp. 332-339, Dec 2001.

[6] J. L. Weston and D. H. Titterton, "Modern Inertial Navigation Technology and its Application," Electronics and Communication Engineering Journal, pp.49-64, April 2000.

[7] C.J.Verplaetse, "Inertial-Optical Motion-Estimating Camera for Electronic Cinematography," M.Sc. Thesis, MIT, Ch.2, June 1994.

[8] C-W. Tan, A. Park, K. Mostov, and P. Varaiya, "Design of Gyroscope-Free Navigation Systems," IEEE Intelligent Transportation Systems Conference Proceedings, pp.286-291, Aug 2001.

[9] J. H. Chen, S. C. Lee, D. B. DeBra, "Gyroscope Free Strapdown Inertial Measurement Unit by Six Linear Accelerometers", Journal of Guidance, Control, and Dynamics, Vol.17, No.2, March-April 1994, pp.286-290.

[10] C. N. Riviere, R. S. Rader, N. V. Thakor, "Adaptive canceling of physiological tremor for improved precision in microsurgery", IEEE Trans Biomned Eng 45(7): 839-46, 1998.
[11] R. Smith, M. Self, and P. Cheeseman, "Estimating Uncertain Spatial Relationships in Robotics", in Autonomous Robot Vehicles, Eds. I.J. Cox and G.T. Wilfong, Springer-Verlag, 1990, pp.167-193. 